\title{
Manufacturing of PolyHIPE-Based Porous Microparticles for Bone Tissue Engineering
}

\author{
T. Paterson, C. Sherborne, C. C. Chen, G. Reilly, F. Claeyssens \\ Kroto Research Institute, Department of Materials Science and Engineering, University of Sheffield.
}

\begin{abstract}
Particle-based systems have great potential as scaffolds for tissue engineering, since they are injectable, avoiding the need for open surgery. When a bone cancer is removed, the void that's formed requires filling with a biomaterial to encourage bone regrowth. Two main methods of filling these voids include using autograft material or bone ceramics. Injectable cell-scaffolds allow keyhole surgery which would be impossible with current methods of treatment. Here we are investigating a method for production of spherical microporous particles of $100-800 \mu \mathrm{m}$. The microporous material is constructed from a HIPE (High Internal Phase Emulsion) via photocuring. The stir-emulsion method produces a wide range of particle sizes and the T-junction fluidic device produces a very narrow range of particle sizes. With both methods it is possible to change the mean particle size and the particle size distribution. The porosity of the particles can be altered independently by the use of temperature during the initial polyHIPE formation. Mesenchymal hES-MPs cells were cultured on particles which had been coated with acrylic acid via plasma deposition. The cells enable the agglomeration of particles into 3D structures with cell growth both into and between particles.
\end{abstract}

Keywords Micro-particle; Mesencymal hES-MP; Particles; PolyHIPE; Tissue Engineering

\section{INTRODUCTION}

The void left from the removal of a bone cancer requires filling with a biomaterial to encourage bone regrowth and to prevent bone resorbtion. Currently the voids require filling with autograft material (which causes donor site morbidity), or bone ceramics (which do not encourage vascularization). We are developing an injectable particle system that will eventually act as a cell carrier whilst actively encouraging angiogenesis through both macro-scale porosity and the release of biomolecules. Allowing the scaffold to be delivered via injection allows the possibility for a minimal surgery approach. Here we are investigating a method for continuous production of spherical microporous particles by stir-emulsion and T-junction fluidic devices. The material is processed as a polyHIPE [1] which allows the production of an extremely porous foam which can be solidified via photocuring. Particles can be produced by curing water in oil in water $(\mathrm{w} / \mathrm{o} / \mathrm{w})$ emulsions. We have also used T-junction fluidics for production of microparticles with highly controlled sizes. Engineering the size of these particles allows tuning of the overall porosity to enhance angiogenesis within these scaffolds, as well as the ability to incorporate regions of specific functionality such as stem cell niches. Mesenchymal hES-MP [2] cells were cultured on particles that had been coated with plasma deposited acrylic acid.

\section{MATERIALS AND METHOdS}

For spherical particle creation, the polyHIPE is mixed into water using an overhead stirrer to form a w/o/w emulsion. The spherical polymer droplets are then cured with a UV lamp. For the T-junction set-up, a small internal diameter $(100 \mu \mathrm{m})$ syringe needle injects the photocurable polyHIPE into a $6 \mathrm{~mm}$ diameter silicone tube containing a continuous stream of $\mathrm{H}_{2} \mathrm{O}$ (sustained via a peristaltic pump) and subsequently cured with a UV lamp (Fig. 1). The set-up produces a continuous stream of particles, which are then collected. PolyHIPE-based structuring allows for a very high degree of porosity, with significant control over the pore size and distribution. Different material coatings have been tested for biocompatibility when cultured with hES-Mp cells. Mesenchymal stem cells are cultured on these particles under static and continuously stirred conditions.

\section{RESULTS}

Spherical particles have been produced with the w/o/w emulsion technique with sizes between 100 and $800 \mu \mathrm{m}$ (Fig. 2). Particles are produced in a distribution of sizes. Spherical particles have been produced with the T-junction fluidic method at 80,200 , and $320 \mu \mathrm{m}$ in size. All particles produced by the fluidics have a size variance of less than $30 \mu \mathrm{m}$. SEM images have been used to analyse the overall particle shape and particle pore size. Histological sectioning was used to investigate internal structure. Plasma-deposited acrylic acid was found to increase biocompatibility when compared to no coatings. No noticeable differences were observed in the

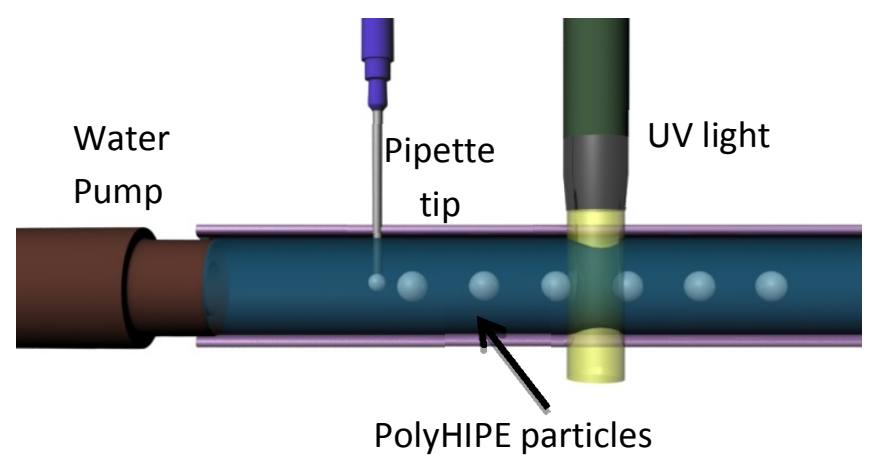

Figure 1. Apparatus used to form uniform polyHIPE particles.

time points between a thin film of the base material compared to the material when formed into a PolyHIPE. Gelatin-coated scaffolds appeared to be similar to noncoated substrate until further tests were carried out to determine that gelatin had not bound to the scaffold. Confocal images of hES-MP cells on PolyHIPE surfaces showed that cells had situated themselves into the pores on the material. hES-MP cells caused the particles to agglomerate together into 3D scaffolds as cells anchored particles together (Fig. 3). 


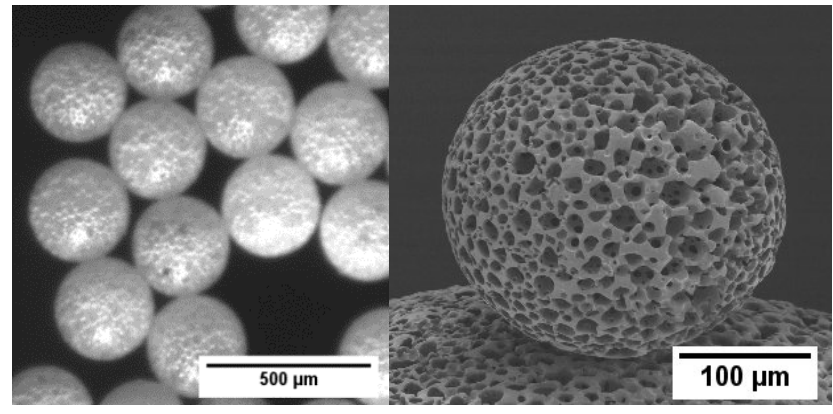

Figure 2. PolyHIPE particles. Left: optical microscope image of 320 $\mu \mathrm{m}$ particles. Right: SEM image of $200 \mu \mathrm{m}$ particle.

\section{DisCUSSION AND CONCLUSIONS}

The T-junction fluidic set-up has enabled the production of very specific sizes, with larger sizes only constrained by the size of the fluidic channel. It is possible to change the size during production, so the option exists to produce a completely uniform mixture of particles, or a mixture containing specific ratios of sizes. The mesenchymal cells grew both into the porosity of the particles and formed anchor points between particles. This allows a larger scaffold to self-form, made from numerous particles. This allows the formation of larger pores between the particles that could enable vascularization. From tests it became apparent that gelatin would not bind to the scaffolds due to their positive surface charge. This is not the case for plasma-deposited acrylic acid, which makes a significant increase to cell proliferation and survival over untreated scaffolds. The binding of the particles by cells shows that it will be possible to create a continuous scaffold from individual particles over a period of time.

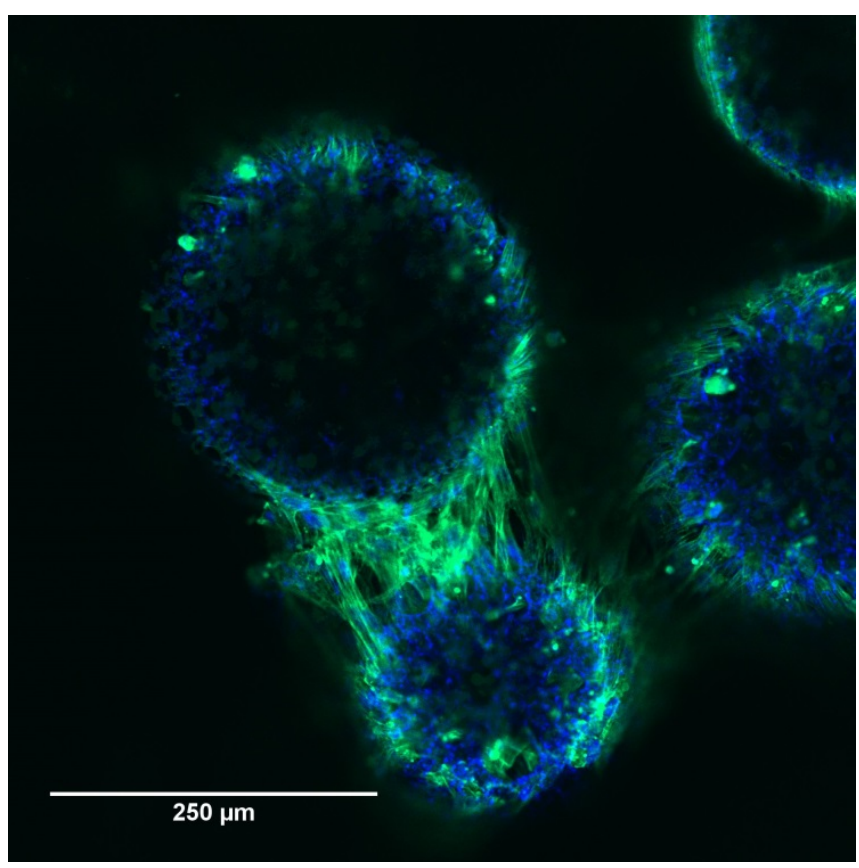

Figure 3. Confocal image of two particles linked by hES-MP cells. Cells stained with DAPI and fitc phalloidin.

\section{ACKNOWLEDGEMENTS}

We acknowledge the EPSRC for funding and Chris Hill for aiding with the SEM imaging. Imaging work was performed at the Kroto Research Institute Confocal Imaging Facility, using the LSM510 Meta upright confocal microscope

\section{REFERENCES}

1. Barbetta, A. and N.R. Cameron, Macromolecules, 2004. 37(9): p. 3188-3201.

2. Karlsson, C., et al., Stem Cell Research, 2009. 3(1): p. 39-50. 